\title{
Closed-form Inverse Determination of Force Excitations for Undamped and Damped Linear-systems Using a Least-squares Approach
}

\author{
A.E. Segall \\ Department of Engineering Science and Mechanics, The Pennsylvania State University, USA
}

Copyright $(2016$ by authors, all rights reserved. Authors agree that this article remains permanently open access under the terms of the Creative Commons Attribution License 4.0 International License

\begin{abstract}
An inverse vibration solution was derived for single DOF systems (undamped and damped) using a method based on the least-squares determination of polynomial coefficients representing the unknown force excitation. Initially, a closed-form and generalized direct-solution to the Vibration Equation was derived using standard polynomials to represent the excitation; while simple polynomials and a single DOF system were chosen to demonstrate the method analytically, more sophisticated functions such as Splines, B-Splines, Basis Functions, and/or Finite-Element analysis can be used with the advocated method. In order to solve the inverse problem, the generalized solutions were then used to enforce measured displacement data and the Levenberg-Marquardt algorithm employed to determine the unknown polynomial coefficients representing the base excitation. Results including random errors added to the data (up to $\pm 5 \%$ ) indicated that the method could be used to determine a time varying excitation; indeed, good agreement was seen between exact and polynomial solution (undamped), as well as an inversely predicted excitation for both damped and undamped systems subjected to a half-sine force history. Given the results, the method appears well suited for complicated scenarios provided the response can be approximated by a polynomial (or more sophisticated functions) and inverse calculations are restricted to the time interval bounding the measured data. In addition, the least-squares inverse approach should be adaptable to more complicated system and excitations by using general purpose finite-element codes. For the current analysis, the least-squares smoothing inherent in the process appears to have helped minimize the influence of measurement errors.
\end{abstract}

Keywords Vibration, Damped, Undamped, Inverse, Excitation

\section{Introduction}

In the study of dynamics where the excitation can involve complex histories, solutions to the ordinary differential equations are readily available for impulse, step, linear, sine, and asymptotic type loading as discussed by Harris [1] and Jacobson et. al [2]. While additional situations can be modeled by using particular solutions for the governing differential equation, the actual excitation often involves empirical data with a time dependency not easily modeled using standard functions.

While inverse approaches for unknown force excitations or system properties have also been considered for vibration problems in the past, they have had limitations or been numerically based. Briefly, Dobson and Rider [3] offer a review of indirect calculation frequency, modal, and finite-element (FEA) models and discuss how they can be used to determine unknown excitation forces. Lancaster et. al [4-5] developed numerical methods based on eigenvalues and eigenvectors to inversely determine vibration system properties such as mass, damping, or stiffness values. On the other hand, Yang [6] derived finite-difference based systems for the determination of unknown exciting forces using least squares methods that did have an advantage in that only one measurement parameter was required. Finally, Doyle [7] developed an inverse method for impact force identification; this analysis involved the use wave propagation data (strain or velocity) and employed a wavelet analysis of the data to determine the relationship between the transform components and unknown force, and then an inverse transform to obtain the desired impacting force history.

While the importance and utility of the numerical and hybrid analytical-numerical simulations cannot be overstated, they do have an unfortunate tendency to produce "black-box" results that are often devoid of any insight into the underlying physics and the relative importance of the variables. Moreover, and perhaps most importantly, all numerical solutions should be verified, bounded, and/or calibrated in some manner to ensure that the model is behaving correctly. Because of these important engineering needs, useful closed-form solutions have been derived for 
both the inverse and direct problems that consider arbitrary force excitations. In addition to the closed formed solutions provided, the proposed method could be applied to more complicated excitations, as well as multi degree-of-freedom systems using a general purpose finite-element approach.

\section{Inverse Solution}

There is clearly a utility for versatile direct and inverse solutions based on relevant excitations in closed form. Fortunately, both can be readily done in a generalized sense for the thermal problem based on temperature or strain (Segall and coworkers [8-9]), as well as vibrations by using generalized vibration solutions developed earlier (Segall [10]). In terms of a direct solution, convolution theory allows the calculation of a system response, $\mathrm{R}(\mathrm{t})$ to an arbitrary force excitation $\Delta(\mathrm{t})$,

$$
\mathrm{R}(\mathrm{t})=\Delta(0) \Phi(\mathrm{t})+\int_{0}^{\mathrm{t}} \frac{\partial \Delta(\tau)}{\partial \tau} \Phi(\mathrm{t}-\tau) \mathrm{d} \tau
$$

where $\mathrm{t}$ represents time, $\tau$ is the dummy integration variable, $\Phi(\mathrm{t})$ is the system response to a unit step excitation, and $\Delta(0)$ represents the excitation at $t=0$. Eq. (1) is often referred to as Duhamel's integral and offers a very practical means for determining the response of a system to an arbitrary excitation in the time domain.

In order to achieve a true degree of generalization for the resulting direct solution given by Eq. (1) while allowing an answer in closed-form, a versatile polynomial containing both integral-and half-order terms can be used to represent the excitation that can now easily include empirical data:

$$
\Delta(\mathrm{t})=\alpha_{0}+\alpha_{1} \mathrm{t}^{\frac{1}{2}}+\alpha_{2} \mathrm{t}+\alpha_{3} \mathrm{t}^{\frac{3}{2}}+\cdot \cdot=\sum_{\mathrm{j}=0}^{\mathrm{n}} \alpha_{\mathrm{j}} \mathrm{t}^{\frac{\mathrm{j}}{2}}
$$

where $\alpha_{j}$ represent polynomial coefficients determined using least-squares or other approximating methods and $\mathrm{n}$ represents the number of terms in the polynomial. It should be noted that for the current demonstrations, integral-and half-order terms were chosen to aid fitting empirical data; other forms of polynomials or equations can be readily used as well. If the excitation can be reasonably fit to this type of polynomial and the unit kernel, $\Phi(\mathrm{t})$ is known (see next sections), then the transient response can be calculated using Equations (1 and 2). However, it should be noted that the proposed method could also potentially use alternate functions (power-law, transcendental, periodic, etc.), as well as piece-wise formulations (Splines, B-Splines, Basis Functions, etc.) if desired.

By using the polynomial given by Eq. (2) with both integral and half-order terms and performing the differentiation of the excitation and subsequent integration of its product with the unit kernel, $\Phi$ (defined in the next sections) required by Eq. (1), the convolution and thus, the generalized solution becomes:

$$
\mathrm{R}(\mathrm{t})=\alpha_{0} \Phi(\mathrm{t})+\int_{0}^{\mathrm{t}} \sum_{\mathrm{j}=1}^{\mathrm{n}} \frac{\mathrm{j}}{2} \alpha_{\mathrm{j}} \tau^{\frac{\mathrm{j}-2}{2}} \Phi(\mathrm{t}-\tau) \mathrm{d} \tau
$$

In this fashion, the product of the unit response and each polynomial term can be integrated individually. With many of the intermediate steps omitted for brevity, the solution now takes on the following generalized form for a polynomial with $\mathrm{n}$ terms:

$$
R(t)=\alpha_{0} \delta_{0}(t)+\frac{1}{k} \sum_{j=1}^{n} \alpha_{j}\left[t^{\frac{j}{2}}-\delta_{j}(t)\right]
$$

where $\delta_{\mathrm{j}}(\mathrm{t})$ represent response functions for the system (dimensionally consistent polynomials of time given in the next sections for undamped and damped systems) and $\mathrm{k}$ is the stiffness. If the excitation is known and can be fit to a polynomial of the form of Eq. (2), then the direct response of the system as a function of time can be readily calculated.

While Eq. (3b) is clearly a direct solution of the problem with a known excitation, namely, $\Delta(\mathrm{t})$, its form that includes a polynomial actually reveals a generalized solution of the corresponding inverse or deconvolution of the problem (Chen et. al [11]) if the response $\mathrm{R}(\mathrm{t})$ is already known. In fact, a generalized inverse solution is possible by fitting measured data, $\mathrm{R}(\mathrm{t})$ obtained at a location remote from the excitation to Eq. (3) with the coefficients determined in a standard least-squares sense (Chen et. al [12]). As such, the inverse problem can then be solved with the determination of the polynomial coefficients for Eq. (2) imbedded in Eq. (3) that satisfy the measured response, $\mathrm{R}(\mathrm{t})$.

\subsection{Undamped Systems}

The first usage of the generalized inverse solution suggested by Eq. (3) involves a commonly encountered situation of a one-degree of freedom system without damping. With initial displacement, velocity, and acceleration at zero, the undamped unit kernel, $\Phi^{\mathrm{U}}(\mathrm{t})$ involves a cosine function of the following form (Harris [1]):

$$
\Phi^{\mathrm{U}}(\mathrm{t})=\frac{\Delta}{\mathrm{k}}\left[1-\cos \left(\omega_{\mathrm{n}} \mathrm{t}\right)\right]
$$

where $\Delta$ is the force and $\mathrm{k}$ is still the stiffness (both equal to unity for this analysis) and $\omega_{\mathrm{n}}$ represents the natural circular frequency for the system. Using this formulation and Eq. (3), the response functions can be defined as:

$$
\delta_{0}^{\mathrm{U}}(\mathrm{t})=\Phi^{\mathrm{U}}(\mathrm{t})
$$

with the first four $2 \mathrm{j}-1$ terms corresponding to the fractional-order polynomial taking the form of:

$$
\delta_{1}^{U}(t)=-\sqrt{\frac{\pi}{2 \omega_{n}}}\left[c(\gamma) \cos \left(\omega_{n} t\right)+s(\gamma) \sin \left(\omega_{n} t\right)\right]
$$




$$
\begin{aligned}
& \delta_{3}^{\mathrm{U}}(\mathrm{t})= \frac{3 \sqrt{\pi}}{\left(2 \omega_{\mathrm{n}}\right)^{\frac{3}{2}}}\left[\mathrm{~s}(\gamma) \cos \left(\omega_{\mathrm{n}} \mathrm{t}\right)-\mathrm{c}(\gamma) \sin \left(\omega_{\mathrm{n}} \mathrm{t}\right)\right] \\
& \delta_{5}^{\mathrm{U}}(\mathrm{t})=-\frac{15 \mathrm{t}^{\frac{1}{2}}}{4 \omega_{\mathrm{n}}^{2}}+ \\
&+\frac{15 \sqrt{\pi}}{\left(2 \omega_{\mathrm{n}}\right)^{\frac{5}{2}}}\left[\mathrm{c}(\gamma) \cos \left(\omega_{\mathrm{n}} \mathrm{t}\right)+\mathrm{s}(\gamma) \sin \left(\omega_{\mathrm{n}} \mathrm{t}\right)\right] \\
& \delta_{7}^{\mathrm{U}}(\mathrm{t})=-\frac{35 \mathrm{t}^{\frac{3}{2}}}{4 \omega_{\mathrm{n}}^{2}}- \\
&-\frac{105 \sqrt{\pi}}{\left(2 \omega_{\mathrm{n}}\right)^{\frac{7}{2}}}\left[\mathrm{~s}(\gamma) \cos \left(\omega_{\mathrm{n}} \mathrm{t}\right)-\mathrm{c}(\gamma) \sin \left(\omega_{\mathrm{n}} \mathrm{t}\right)\right]
\end{aligned}
$$

The first four $2 \mathrm{j}$ terms corresponding to the integral order portion of the polynomial are:

$$
\begin{gathered}
\delta_{2}^{\mathrm{U}}(\mathrm{t})=-\frac{\sin \left(\omega_{\mathrm{n}} \mathrm{t}\right)}{\omega_{\mathrm{n}}} \\
\delta_{4}^{\mathrm{U}}(\mathrm{t})=-\frac{2}{\omega_{\mathrm{n}}^{2}}+\frac{2 \cos \left(\omega_{\mathrm{n}} \mathrm{t}\right)}{\omega_{\mathrm{n}}^{2}} \\
\delta_{6}^{\mathrm{U}}(\mathrm{t})=-\frac{6 \mathrm{t}}{\omega_{\mathrm{n}}^{2}}+\frac{6 \sin \left(\omega_{\mathrm{n}} \mathrm{t}\right)}{\omega_{\mathrm{n}}^{3}} \\
\delta_{8}^{\mathrm{U}}(\mathrm{t})=-\frac{12 \mathrm{t}^{2}}{\omega_{\mathrm{n}}^{2}}+\frac{24}{\omega_{\mathrm{n}}^{4}}-\frac{24 \cos \left(\omega_{\mathrm{n}} \mathrm{t}\right)}{\omega_{\mathrm{n}}^{4}}
\end{gathered}
$$

An inspection of Equations 5b-5e reveals an underlying pattern of descending sub-polynomials for each fractional order term:

$$
\begin{aligned}
& \delta_{2 j-1}^{\mathrm{U}}(\mathrm{t})=(-1)^{\operatorname{Int}\left(\frac{\mathrm{j}+2}{4}\right)+1} \frac{\sqrt{\pi}}{\left(2 \omega_{\mathrm{n}}\right)^{\frac{2 \mathrm{j}-1}{2}}}\left|\beta\left(\operatorname{Int}\left(\frac{2 \mathrm{j}-1}{4}\right)+1,2 \mathrm{j}-1\right)\right| \Psi_{2 \mathrm{j}-1} \\
& +\sum_{\mathrm{i}=1}^{\operatorname{Int}\left(\frac{2 \mathrm{j}-1}{4}\right)}(-1)^{\mathrm{i}+2} \frac{\mathrm{t}^{\frac{2 j-1-4 \mathrm{i}}{2}}}{\left(\omega_{\mathrm{n}}\right)^{2 \mathrm{i}}} \beta(\mathrm{i}, \mathrm{j})
\end{aligned}
$$

Equations $5 \mathrm{f}-5 \mathrm{i}$ also reveal an underlying pattern of descending sub-polynomials for each integral order term:

$$
\begin{aligned}
& \delta_{2 \mathrm{j}}^{\mathrm{U}}(\mathrm{t})=(-1)^{\mathrm{j}} \frac{\mathrm{j} !}{\omega_{\mathrm{n}}^{\mathrm{j}}} \sin \left(\omega_{\mathrm{n}} \mathrm{t}+\frac{\pi(\mathrm{j}-1)}{2}\right)+ \\
& +\sum_{\mathrm{i}=1}^{\operatorname{Int}\left(\frac{\mathrm{j}}{2}\right)}(-1)^{\mathrm{i}+1} \frac{\mathrm{t}^{\mathrm{j}-2 \mathrm{i}}}{\omega_{\mathrm{n}}^{2 \mathrm{i}}} \chi(2 \mathrm{i}, \mathrm{j})
\end{aligned}
$$

where the various recurring functions are defined as:

$$
\psi_{2 \mathrm{j}-1}=\left\{\begin{array}{cc}
\mathrm{c}(\gamma) \cos (\omega \mathrm{t})+\mathrm{s}(\gamma) \sin (\omega \mathrm{t}) & 2 \mathrm{j}-1=1,5,9 \ldots \\
\mathrm{s}(\gamma) \cos (\omega \mathrm{t})-\mathrm{c}(\gamma) \sin (\omega \mathrm{t}) & 2 \mathrm{j}-1=3,7,11 \ldots
\end{array}\right.
$$

and

$$
\gamma=\sqrt{\frac{2 \mathrm{t} \omega_{\mathrm{n}}}{\pi}}
$$

with the natural circular frequency for the system

$$
\omega_{\mathrm{n}}=\sqrt{\frac{\mathrm{k}}{\mathrm{m}}}
$$

and $\mathrm{m}$ representing the mass of the system. Additionally, $c(\gamma)$ and $s(\gamma)$ represent the cosine and sine based Fresnel Integrals, respectively while the remaining terms are defined as follows:

$$
\beta(\eta, \lambda)=\frac{2^{\eta} \Gamma(\lambda / 2+1)}{\Gamma(\lambda / 2+1-\eta)}
$$

and

$$
\chi(\eta, \lambda)=\frac{\lambda !}{(\lambda-\eta) !}
$$

\subsection{Damped Systems}

For a linear system with viscous damping, the damped unit kernel, $\Phi^{\mathrm{D}}(\mathrm{t})$ invokes the following form (Harris [1]):

$$
\Phi^{\mathrm{D}}(\mathrm{t})=\frac{\Delta}{\mathrm{k}}\left\{1-\mathrm{e}^{-\zeta \omega_{\mathrm{n}} \mathrm{t}}\left[\cos \left(\omega_{\mathrm{d}} \mathrm{t}\right)+\frac{\zeta}{\sqrt{1-\zeta^{2}}} \sin \left(\omega_{\mathrm{d}} \mathrm{t}\right)\right]\right\}
$$

where $\Delta$ is again the force and $\mathrm{k}$ the stiffness (both equal to unity for all calculations), $\zeta$ is the damping ratio, and $\omega_{\mathrm{d}}$ represents the damped natural frequency for the system:

$$
\omega_{\mathrm{d}}=\omega_{\mathrm{n}} \sqrt{1-\zeta^{2}}
$$

As before, the first term of the generalized direct solution may be defined as the unit response such that:

$$
\delta_{0}^{\mathrm{D}}(\mathrm{t})=\Phi^{\mathrm{D}}(\mathrm{t})
$$

Given the form of the unit step response, the required integration with the half- and integral-order polynomial terms could not produce an analytical solution involving standard functions. As such, the individual response functions must be represented in their integral form:

$$
\delta_{j}^{D}(t)=\alpha_{j} \int_{0}^{t} \frac{j}{2} \tau^{\frac{j-2}{2}} \bar{\Phi}^{D}(t-\tau) d \tau
$$


where the modified unit response, $\bar{\Phi}^{\mathrm{D}}(\mathrm{t})$ is defined as follows:

$$
\bar{\Phi}^{\mathrm{D}}(\mathrm{t})=\mathrm{e}^{-\zeta \omega_{\mathrm{n}} \mathrm{t}}\left[\cos \left(\omega_{\mathrm{d}} \mathrm{t}\right)+\frac{\zeta}{\sqrt{1-\zeta^{2}}} \sin \left(\omega_{\mathrm{d}} \mathrm{t}\right)\right]
$$

For $\mathrm{j}=1,2,3 \ldots \mathrm{n}$ and using the definitions above, the generalized response given by Eq. (3) must ultimately be evaluated via quadrature for both the direct and inverse solutions:

\section{Results and Discussion}

For the initial verification calculations, the accuracy of the vibration response functions were compared to existing solutions for an undamped single-degree of freedom system using MathCad software (PTC Inc., Needham MA); the least-squares determination of the unknown coefficients was accomplished via the Levenberg-Marquardt algorithm. For this comparison, a half-sine function shown in Figure (1) was chosen for the excitation with $\pm 5 \%$ random error introduced. Although not a perfect fit as indicated by the oscillations around various data points, an 8-term polynomial was shown to be capable of approximating the excitation over the entire time period; a significantly improved polynomial fit is possible if the analysis is restricted to the time interval defined by the first period of $0 \leq \mathrm{t} \leq \pi$. Despite the "waviness" of the polynomial and its apparent inability to match a sharp corner, very good agreement was observed when the simulation for the undamped case was compared to an existing closed-form solution (Harris [1]) as shown by Figure (2), even with the $\pm 5 \%$ error randomly introduced.

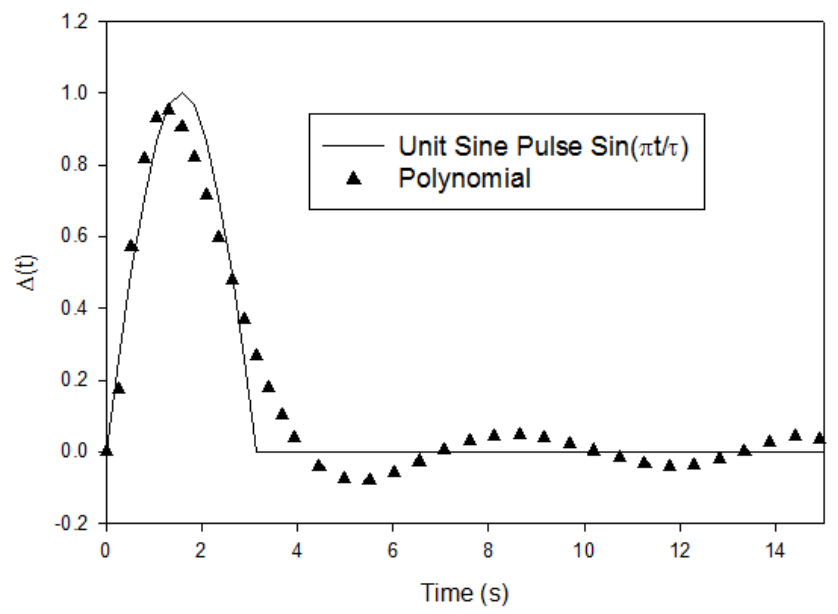

Figure 1. Comparison of the "goodness-of-fit" (and inverse estimation) of a polynomial and the half-sine excitation.

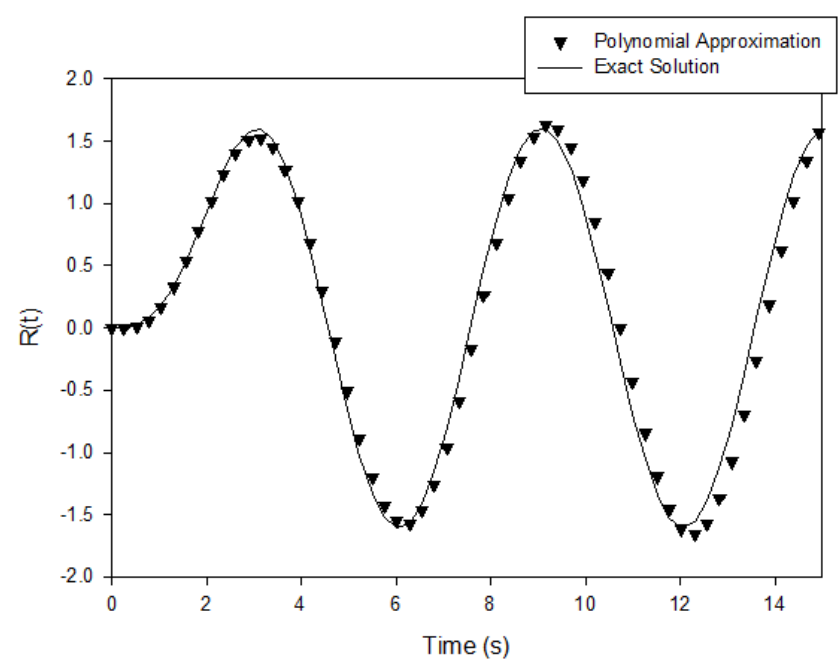

Figure 2. Comparison of exact and polynomial based predictions of the undamped transient response of a single degree of freedom system to a half-sine excitation.

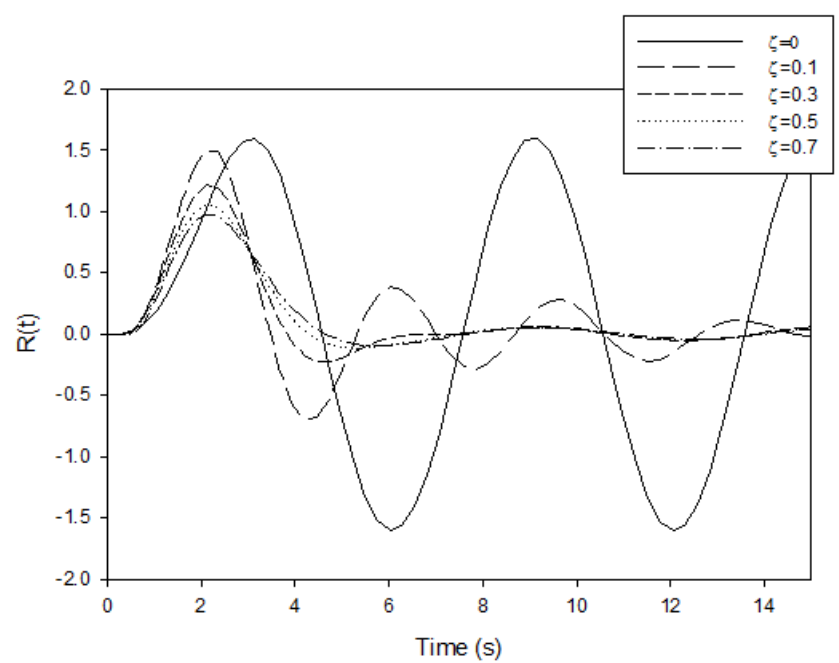

Figure 3. Polynomial based predictions of the damped transient response of a single degree of freedom system to a half-sine excitation for various damping ratios.

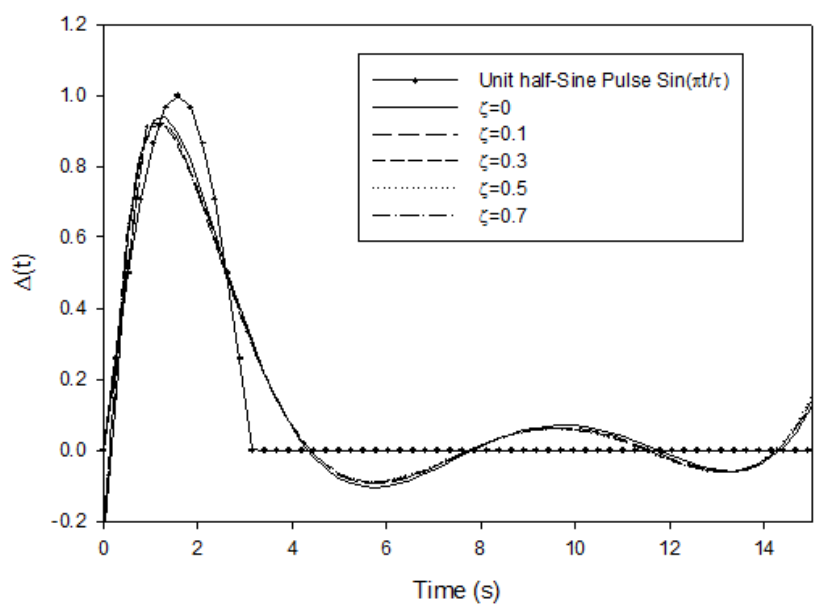

Figure 4. Comparison of inverse estimates of the half-sine excitation for various damping ratios and the generalized responses shown in Figure 3. 
The approximated response shown in Figure (2) was then used as input data for the deconvolution of the excitation. For this process, the calculated response data was then fit to Eq. (3b) and the unknown polynomial coefficients determined. Once the coefficients were determined, Eq. (2) was used to estimate the unknown excitation. The resulting polynomial was virtually identical to the half-sine input, as well as the original approximating polynomial shown in Figure 1 (and is in-fact, the inverse prediction shown in Figure 4 for $\zeta=0$ ), even with the $\pm 5 \%$ error randomly introduced. Thus the proposed least-squares method was shown to be capable of producing a very good estimation of the excitation.

Using the same half-sine excitation and overall approach, the damped case was also estimated using quadrature. Figure 3 shows the effects of the increasing damping ration on the overall response of the system. As with the earlier simulations, the calculated response data was then fit to Eq. (3b) and the unknown polynomial coefficients determined, this time for various damping ratios. As shown by Figure 4, the resulting predictions were all nearly identical to each other, as well as to the original approximating polynomial to the half-sine input, even with the $\pm 5 \%$ error randomly introduced. Thus, the proposed least-squares method again provided a very good estimation of the excitation even with various levels of damping considered.

While the results were very promising and clearly demonstrate the potential of the proposed method, care should always be exercised to ensure that the polynomial reasonably describes the excitation over the desired time interval. Moreover, any calculations outside the data interval used to determine $\Delta(t)$ will most likely produce erroneous results as the polynomial may begin to go astray. Since these are all inherent pitfalls of curve fitting, the methods described in this study should be used carefully and not to extrapolate the temporal behavior of the system beyond the boundaries of the data. Nonetheless, the least-squares smoothing inherent in the process appears to have helped to minimize the influence of random measurement errors introduced in the analysis.

Given the results, the analytical solutions appears capable of handling relatively complicated scenarios provided the response can be approximated by a polynomial and analysis is restricted to the time interval bounding the data. As already mentioned, simple polynomials were chosen for the analysis to facilitate closed-form solutions and demonstrate the efficacy of the proposed method; the use of more sophisticated functions should also be explored. Although their closed-form nature will begin to be obscured, the derived solutions could be easily modified to accommodate Spline type curves. In fact, algorithms based on constrained B-Splines employing least-squares smoothing [13] make sense since they would allow a more precise fit of the experimental data while retaining smoothing capabilities that may be helpful with inherent errors in the data.

Finally, while the method was demonstrated in closed-form for a simple polynomial, it can also be used for more complex geometries and multiple DOF systems. In fact, finite-element (or analogous) numerical calculations could be used to approximate a unit response for just about any configuration. In turn, this unit approximation could then be fit to a function (anything from a simple polynomial to a multi-dimensional form of Eqs. (4 or 13)) and then used analytically as described in this paper. Given the relative ease at which finite-elements can be used to model complex situations and geometries, this could prove to be an efficient means to solve the inverse problem for very complicated systems.

\section{Conclusions}

Convolution theory along with a versatile polynomial composed of half- and integral-order terms was used to model the response of various systems to arbitrary force excitations. Very good agreement was then seen when the newly derived relationships were compared to existing direct solutions for undamped and damped, single-degree of freedom systems. When the calculated response was used as input (i.e., as if measured) data, the method applied in a least-squares sense was also shown to be able to determine the underlying excitation and solve the inverse problem. Although approximate in nature because of the use of a polynomial, the method allows the simulation of complex excitations not easily modeled using standard functions. Higher order polynomials, Splines, and/or different Basis functions can also be used provided the required integrations are possible in either closed-form or via quadrature, and the solution algorithms can converge to an answer. However, it is recommended that the polynomial order be kept as low as possible to avoid additional "wiggle" and/or convergence issues. The derived expression can and should be used to verify, bound, and/or calibrate solutions based on more sophisticated numerical methods when excitations involving complex temporal relationships are involved. Moreover, the proposed approach along with finite-element (or analogous) calculations could also be used to approximate a unit response for just about any configuration, thus providing a relatively easy method for determining unknown excitations based on measured data. Hence, the methods just proposed outline a procedure where inverse predictions may be possible for complex situations by using commercially available, generalized-packages without the need for specialized computer codes or methods.

\section{REFERENCES}

[1] Harris, C.M., 1961, Shock \& Vibration Handbook, McGraw-Hill Book Company, New York.

[2] Jacobson, L.S. and Ayre, R.S., 1952, "A Comparative Study of Pulse and Step-Type Loads on Simple Vibratory Systems," 
Technical Report N16.

[3] Dobsen, B.J. and Rider, E., 1990, "A review of the Indirect Calculation of Excitation Forces from Measured Structural Response Data, Proc. Inst. Mech. Engrs., 204, pp.69-75.

[4] Lancaster, P. and Maroulas, J, "Inverse Eigenvalue Problems for Damped Vibrating Systems," Journal of Mathematical Analysis and Applications, 123, pp. 238-261, 1987.

[5] Lancaster, P. and Prells, U, "Inverse Problems for Damped Vibrating Systems," Journal of Sound and Vibration, 283, pp. 891-914, 2005.

[6] Yang, C.Y., 1996, "Solution of an Inverse Vibration Problem using a Linear Least Squares method," Applied Math. Modelling, 20, pp. 785-788.

[7] Doyle, J.F., 1997, “A Wavelet Deconvolution Method for Impact Forces," Experimental Mechanics, 37, pp. 403-408.

[8] Segall, A.E., "Inverse Solutions for Determining Arbitrary Boundary-Conditions using a Least-Squares Approach," Technical note in the ASME Journal of Heat Transfer, 127, pp. 1403-1405, December 2005.
[9] Segall, A.E, Engels, D., and Hirsh, A. "Transient Surface Strains and the Deconvolution of Thermoelastic States and Boundary Conditions," ASME Journal of Pressure Vessel Technology, 131 [1], pp. 011201-1-011201-9, February 2009.

[10] Segall A.E., "Useful Polynomials for the Transient Response of Solids under Mechanical and Thermal Excitations," International Journal of Mechanical Sciences, Vol. 44, No. 4, pp. 809-823, 2002.

[11] L. Y. Chen, J. T. Chen, H.-K. Hong and C. H. Chen, 1995, Application of Cesaro Mean and the L-curve for the Deconvolution Problem, Soil Dynamics and Earthquake Engineering, Vol.14, No.5, pp.361-373.

[12] J. T. Chen, C. F. Lee and S. Y. Lin, 2002, A new point of view for the polar decomposition using singular value decomposition, Int. J. Comp. Numer. Anal. Appl., Vol.2, No.3, pp. 257-264

[13] Hanson, R.J., "Constrained Least Squares Curve Fitting to Discrete Data Using B-Splines--A Users Guide," Sandia Report SAND78-1291, 1979. 